\title{
Physical Interpretation of Inverse Dynamics using Bicausal Bond Graphs
}

\author{
Peter J Gawthrop ${ }^{a}$ \\ ${ }^{\mathrm{a}}$ Centre for Systems and Control and Department of Mechanical Engineering, \\ University of Glasgow, GLASGOW. G12 8QQ Scotland. \\ P.Gawthrop@eng.gla.ac.uk \\ An earlier version of this paper was accepted for the Bond Graph Digest - an \\ electronic journal which no longer seems to be in existence
}

\begin{abstract}
A physical interpretation of the inverse dynamics of linear and nonlinear systems is given in terms of the bond graph of the inverse system. It is argued that this interpretation yields physical insight to guide the control engineer.

Examples are drawn from both robotic and process systems.
\end{abstract}

Key words: Bond graphs; system inversion; inverse dynamics; control 


\section{Introduction}

It has been argued by Sharon et al. (1991) that control system design could, and should, be based on physical system insight. Bond graphs (Karnopp and Rosenberg, 1975; Thoma, 1975; Wellstead, 1979; Rosenberg and Karnopp, 1983; Karnopp et al., 1990; Gawthrop and Smith, 1996) provide a high-level modelling language for describing dynamic systems in a graphical form which retains such physical insight. For these reasons, physical-model-based control using bond graphs has been suggested by Karnopp (1979), Roberts et al. (1995), Karnopp (1995) and Gawthrop (1995b) as an approach to control system design. This paper focuses on one aspect of this: the physical interpretation of zero dynamics (Isidori, 1995) (system zeros in the case of linear systems) in the context of control system design ${ }^{1}$.

This concept is, in turn, related to a classical problem in control (particularly in the field of Process Engineering): the determination of the configuration of the control system in the sense of determining inputs, outputs and feedforward terms for the purposes of effective control. This is called the control loop pairing problem. This is not primarily to do with system parameters, but rather with system structure; thus this is largely a qualitative, as opposed to a quantitative, problem. Bond graphs make a clear distinction between system structure (as represented by the bond graph) and numerical parameters; therefore bond graphs are an appropriate tool in this context. There is a particular interest at the moment in including control considerations at the process design stage (see, for example, Ponton and Laing (1993), Laing (1995), Wolff (1994) and the references therein) to complement the relatively well established conceptual design on process plant alone as, for example discussed by Douglas (1988).

There are many transfer function based approaches to the control configuration problem including the relative gain array of Bristol (1966) and a number of singular value decomposition based methods such as that of Havre and Skogestad (1996). Following the "Design in the Physical Domain" approach of Sharon et al. (1991), it is argued here that it is better to stick to the physical model itself than to immediately use an abstraction, such as a transfer function; bond graphs provide an appropriate physical domain representation. A bond graph interpretation of the relative gain array, and related indicators, has already been given by Gawthrop et al. (1999).

The approach taken here is to investigate the possibility of ideal control; that is, is it possible in principle to set system outputs to desired values and deduce the corresponding inputs: and this leads to the notion of system in-

$\overline{1}$ Zero dynamics and related issues are discussed further in Section 2. 
version (or partial inversion) with respect to input-output pairs (Gawthrop and Smith, 1996; Ngwompo, 1997; Ngwompo et al., 1996). As discussed by Gawthrop (1995a) bicausal bond graphs have an important role in investigating the inverses of a system described by bond graphs; in particular, the bond graph of the system inverse is often best represented by a bicausal bond graph. A contribution of this paper is to give a physical interpretation of zeros (zero dynamics in the nonlinear case) in the context of bond graphs.

The approach differs from the work of Huang and Youcef-Toumi (1999) and the earlier work of Gawthrop and Smith (1996) in that the concept of bicausality is used to represent the causal implications of inversion (Ngwompo, 1997; Ngwompo et al., 1996). Without bicausality, the bond graph does not completely represent the the inverse system and the resulting zero dynamics due to the need to solve an additional set of algebraic equations which are not explicitly represented in the bond graph.

Building on the basic ideas of bond graph based control presented by Gawthrop (1995b), this paper provides an alternative approach to control system configuration which is based on bond graph inversion and bicausal bond graphs.To support this, extensions of the bicausal bond graph theory to systems with modulation are given.

Because of the multi-disciplinary nature of bond graphs, the method is applicable to a range of engineering systems. Hence the ideas are illustrated using both a mechanical and process engineering examples. The Figures are generated using a bicausal version of a set of Model Transformation Tools Gawthrop (1999) which is currently under development.

The outline of the paper follows. Section 2 discusses the role of zero dynamics in determining the controllability (in a loose sense) of a system. Section 3 gives the background theory relating to bicausal bond graphs and Section 4 shows how bicausal bond graphs can be used to give the bond graph of the inverse of a dynamic system. Section 5 gives a mechanical (robotic) example due to Sharon (1988) illustrating lightly-damped zeros. Section 6 gives a physical interpretation of unstable linear zero dynamics. Section 7 takes a detailed look at control loop pairing for a two-input two-output nonlinear model of a non-isothermal chemical reactor and gives a physical interpretation of the (nonlinear) zero dynamics.

Some of this material has already appeared in conference papers (Gawthrop, 1995a; Gawthrop, 1997). 


\section{System Controllability and Zero Dynamics}

The controllability of a system has at least two meanings:

- a strict state-space concept which in the linear case corresponds to the nonsingularity of a certain matrix (Kwakernaak and Sivan, 1972; Kailath, 1980) and

- a somewhat broader (and practically more useful) concept developed over the years in the process engineering community to do with how difficult a system is to control.

It is the latter meaning that is used here.

There are at least two reasons why a particular input/output pair of a linear system described by a rational transfer function may be hard to control:

(1) the relative degree ${ }^{2} \rho$ of the corresponding transfer function (number of poles - number of zeros) is large,

(2) the zeros of the corresponding $m$ th order transfer function are

(a) lightly damped or

(b) in the right-half plane.

This fundamental result has been known to control engineers for some time. Some relevant textbooks (old and new) are those of Bode (1945), Horowitz (1963), Maciejowski (1989) and Skogestad and Postlethwaite (1996).

In the linear case, both reasons are related to system zeros; in the first case their number and in the second case their location. Although the dynamics giving rise to system poles are system poles are readily identified from the system bond graph (they arise from the $\mathbf{C}^{3}$ and $\mathbf{I}$ elements in integral causality), the dynamics giving rise to the zeros cannot be readily identified. However, the (finite) zeros of a system correspond to the poles of the system inverse. Hence the system zeros can be related to the $\mathbf{C}$ and $\mathbf{I}$ elements in integral causality in the bond graph of the inverse system - this is the core idea of this paper.

The corresponding problems (along with others) also arise in the case of nonlinear systems. Although the concept of poles and zeros are no longer applicable, the concepts of relative degree and the system inverse are still relevant. In particular, the concept of zero dynamics, the dynamics of the system inverse, is a key component of nonlinear systems theory. The textbooks of Isidori (1995)

$\overline{2}$ The relative degree of a transfer function is the difference of the degrees of the denominator polynomial and the numerator polynomial.

3 We use bold text to indicate bond graph components throughout the paper. 
and Marino and Tomei (1995) give an in-depth treatment of such issues in the context of control system design.

To get a useful theory of "Design in the Physical Domain", it is necessary to get a physical interpretation of these concepts, which are both associated with the system zeros (zero dynamics in the nonlinear case) the main idea in this paper is that this is best achieved by examining the bond graph of the inverse system.

As discussed in Section 4, the bond graph of the system inverse (with respect to given input-output pair) is the same as the system bond graph, but with changed causal strokes. Usually, this changed causality forces derivative causality on some of the dynamic ( $\mathbf{C}$ or $\mathbf{I}$ ) components which will no longer contribute to the system state. The following information about the dynamic system connecting the particular input/output pair then follows directly by comparing the bond graphs of the system and its inverse:

- the relative order $\rho$ of the system is the number of dynamic ( $\mathbf{C}$ or $\mathbf{I}$ ) components which change from integral to derivative causality,

- the zero dynamics of the system are formed by the $m$ dynamic components of the inverse system ( $\mathbf{C}$ or $\mathbf{I}$ ) which retain integral causality; the order of the inverse dynamics corresponds to the number of these components.

This paper focuses on the properties of the individual control loops of a multivariable system; the corresponding properties are dependent upon the assumptions made about the remaining control loops. There are three possibilities for each of the other control loops:

(1) the control loop is closed by a controller of defined structure;

(2) the control loop is open;

(3) the control loop is closed by an ideal controller - the corresponding pair is inverted.

Possibility 1 is examined in Section 5 and possibilities 2 and 3 are examined in Section 7.

\section{Bicausal Bond Graphs}

Bicausal bond graphs were introduced by Gawthrop (1995a) to give a foundation for deriving system properties relating to

- system inversion

- state estimation and

- parameter estimation 
directly from the system bond graph. This paper expands on the first of these: system inversion.

This section provides a brief review of the material and extends the concepts to include modulation and active bonds. Some of the techniques are related to those introduced by Cornet and Lorenz (1989). Further developments have been reported by Ngwompo et al. (1996) and Ngwompo et al. (1997).

\subsection{Bicausal bonds}

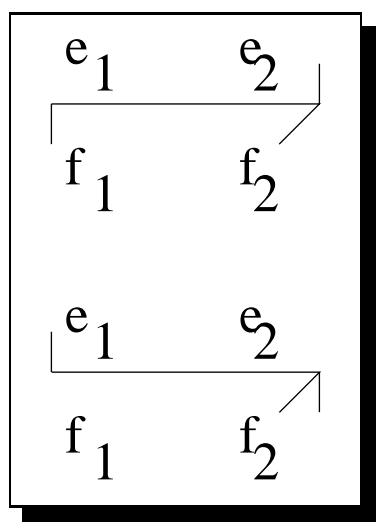

Fig. 1. Bicausal bonds

As discussed by Gawthrop (1995a), the two bicausal bonds appearing in Figure 1 correspond to the following pairs of equations respectively:

$$
\begin{array}{ll}
e_{2}:=e_{1} & f_{2}:=f_{1} \\
e_{1}:=e_{2} & f_{1}:=f_{2}
\end{array}
$$

In other words, the notation is such that:

- a causal half stroke on the flow side of the bond (we adopt the common convention that the half arrow is on the flow side of the bond) implies that flow is imposed on the variable associated with the far end of the bond (the variable associated with the far end of the bond is on the left-hand side of the assignment statement) and

- a causal half stroke on the effort side of the bond implies that effort is imposed on the variable associated with the near end of the bond (the variable associated with the near end of the bond is on the left-hand side of the assignment statement).

This notation has the happy result that when the causal half strokes are on the same end of the bond then they have the same meaning as the corresponding 
causal (full) stroke of conventional bond graph notation.

\subsection{Bicausal junctions}

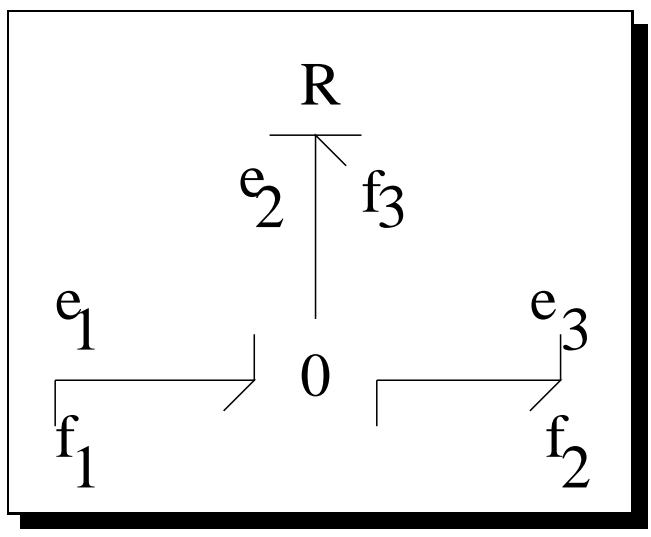

Fig. 2. A bicausal junction

As discussed by Gawthrop (1995a), bicausality can be propagated though junctions; Figure 2 shows a typical situation. The $\mathrm{R}$ component is assumed known and thus is unicausal. An effort $e_{1}$ impinges on the bond forcing causality on the $\mathrm{R}$ component. The flows $f_{1}$ and $f_{3}$ thus imply $f_{2}$ as shown.

\subsection{Source-sensor components}

Traditionally, the $S_{e}$ and $S_{f}$ components have a special status amongst bond graph components in that the corresponding causality is irrevocably fixed; this causes problems in the context of this paper. In addition, they are sometimes used to represent the measurement of the corresponding flow and effort respectively.

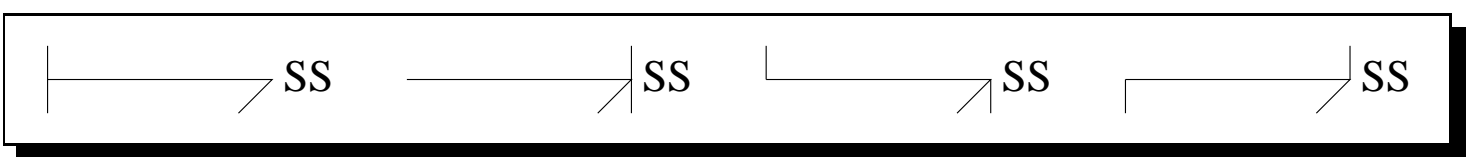

Fig. 3. Source sensors

These components are not used in this paper, but, as discussed by Gawthrop (1995a) they are replaced by the source-sensor (SS) component of Figure 3.

Figure 3 shows the four possible bicausal versions. In order they imply:

(1) effort source/flow sensor

(2) flow source/effort sensor

(3) flow and effort source 
(4) flow and effort sensor

\subsection{Active bonds}

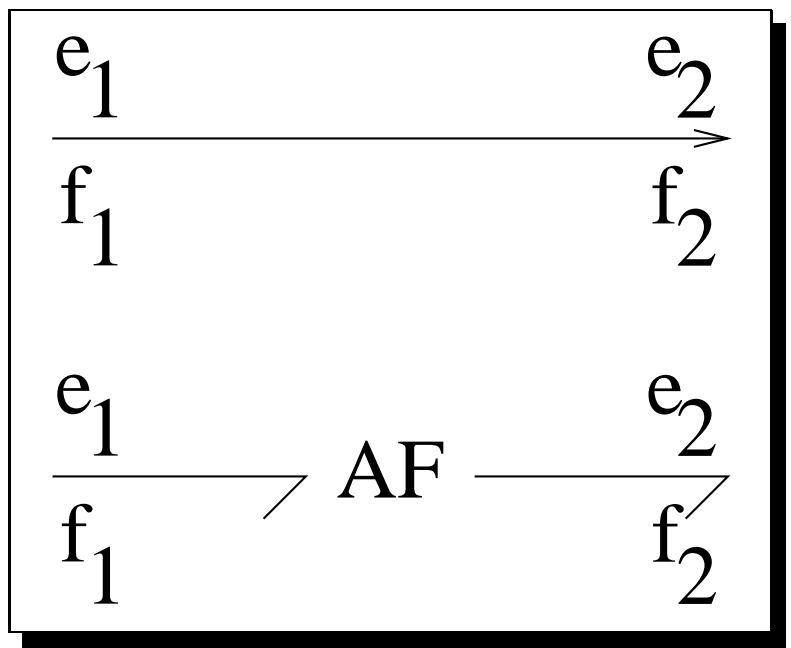

Fig. 4. Active bonds

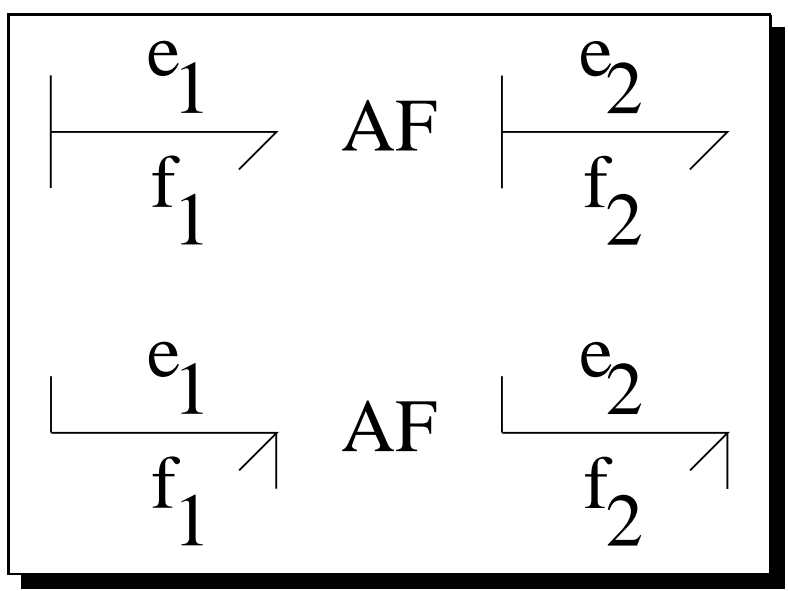

Fig. 5. Amplifier causality

Active bonds are necessary to model many systems, including process systems. This section extends the bicausal bond graph idea to include active bonds.

Unfortunately, the standard notation for active bonds does not lend itself to the bicausal notation for a number of reasons:

- (uni) causal strokes are not usually added to active bonds;

- the lack of a half-stroke makes the bicausal notation used here ambiguous and

- the active bond variable type (effort or flow) depends on the component to which the non-arrow end is connected. 
For these reasons, and following Gawthrop and Smith (1996), active bonds are reinterpreted as two-port amplifier components together with a pair of power bonds.

Figure 4 shows a flow-carrying active bond interpreted as the ideal two-port unit gain flow amplifier AF component (Gawthrop and Smith, 1996). In its usual setting, this component is described by the equations:

$$
e_{1}:=0 \quad f_{2}:=f_{1}
$$

However, if the amplifier output (flow) is known, the corresponding assignment statement for the input (flow) can be written down to give the alternative pair of equations:

$$
e_{1}:=0 \quad f_{1}:=f_{2}
$$

Equations 3 and 4 correspond to the upper and lower causal patterns of Figure 5 respectively.

An effort-carrying active bond can be modelled in the same sort of way using the effort amplifier AE component.

\subsection{Flow-modulated resistors}

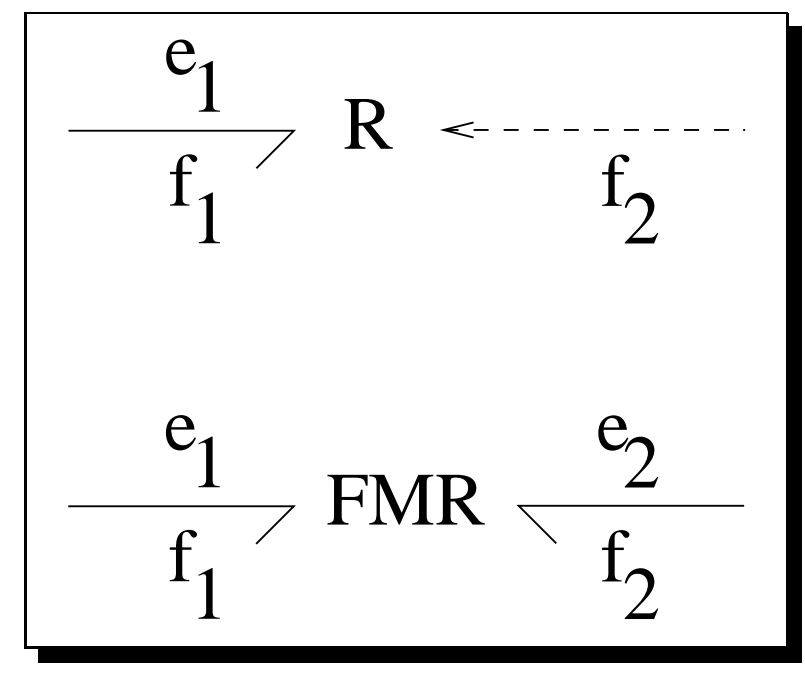

Fig. 6. A Flow-modulated resistor

Flow-modulated resistors are necessary to model many systems, including process systems. This section extends the bicausal bond graph idea to include flow-modulated resistors. Figure 6 shows the modulated resistor introduced by MacKenzie et al. (1993) (see also Gawthrop and Smith (1996)) in the context of modeling chemical processes. In this paper, this modulated resistor is 


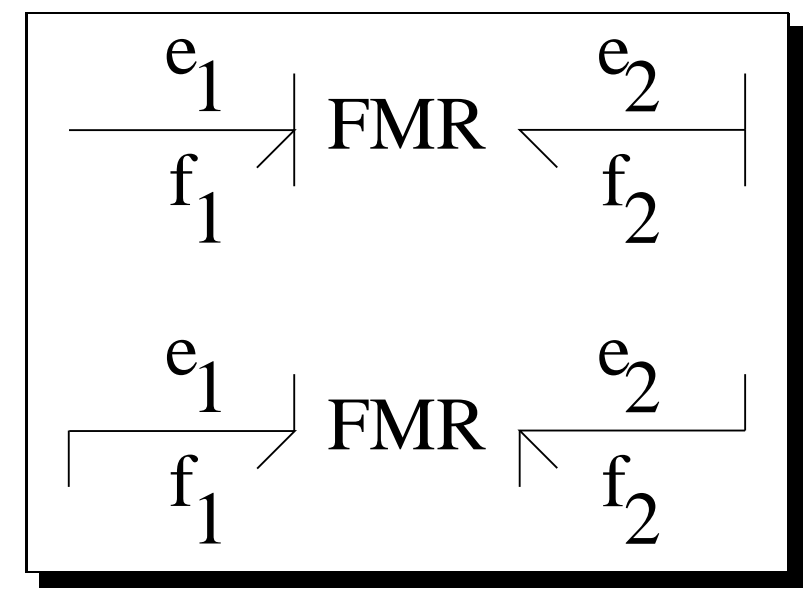

Fig. 7. A Flow-modulated resistor with causality

replaced by the two-port flow-modulated resistor component (FMR) in the lower part of Figure 7.

In its usual setting, this component is described by the equations:

$$
f_{1}:=k f_{2} e_{1} \quad e_{2}:=0
$$

However, if both covariables on port 1 are known, the corresponding assignment statement for the input (flow) can be written down to give the alternative pair of equations:

$$
f_{2}:=\frac{f_{1}}{k e_{1}} \quad e_{2}:=0
$$

Equations 5 and 6 correspond to the upper and lower causal patterns of Figure 7 .

The FMR component is used in the example of Section 7.

\section{System Inversion}

System inversion is discussed by Gawthrop and Smith (1996) and shown to correspond to the reversal of causality on SS components. However, the method presented there implies the use of constraint equations not directly represented on the bond graph; the resultant bond graph cannot be easily used to extract qualitative information about the system inverse.

The introduction of bicausal bond graphs by Gawthrop (1995a) allows all of the equations describing the system inverse to be directly represented on the bond graph of the inverse (an exception to this arises if there are causal loops associated with the system). 


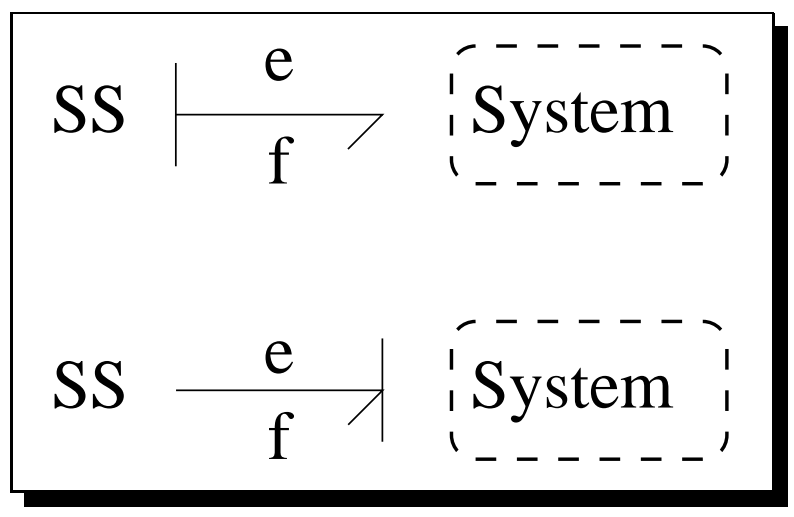

Fig. 8. Inversion of system with colocated input-output

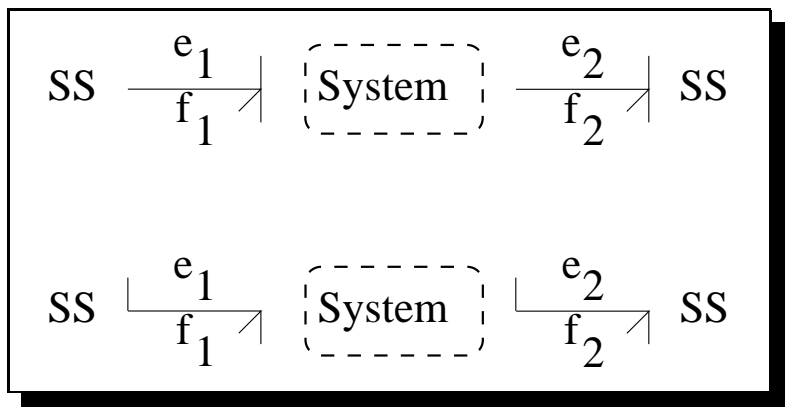

Fig. 9. Inversion of system with non-colocated input-output

The SS component introduced by Gawthrop and Smith (1992) and discussed further in Section 3 is a convenient replacement for the standard $\mathbf{S}_{e}$ and $\mathbf{S}_{f}$ components and provide the input and output ports of a system. The notion of input and output can be simply altered by moving the causal strokes appropriately.

The upper part of Figure 8 refers to a system with input $f$ and output e. This system has a colocated input and output in the sense that the input and output are bond covariables. The lower part of Figure 8 refers to the inverse system in the sense that the roles of $e$ and $f$ are reversed. This inversion is expressed by standard bond graph notation.

The upper part of Figure 9 refers to a system with two inputs $e_{1}$ and $f_{2}$ and two outputs $e_{2}$ and $f_{1}$. This system has a non-colocated inputs output pair $e_{1}$ and $e_{2}$ in the sense that this input and output pair are not bond covariables. The lower part of Figure 8 refers to the inverse system with respect to the pair $e_{1} e_{2}$ in the sense that the roles of $e_{1}$ and $e_{2}$ are reversed. This inversion is expressed by the bicausal bond graph notation.

Sections 5, 6 and 7 gives examples of this process and how it can be used to determine qualitative information about the system inverse. 


\section{Example: Macro-micro Manipulator}

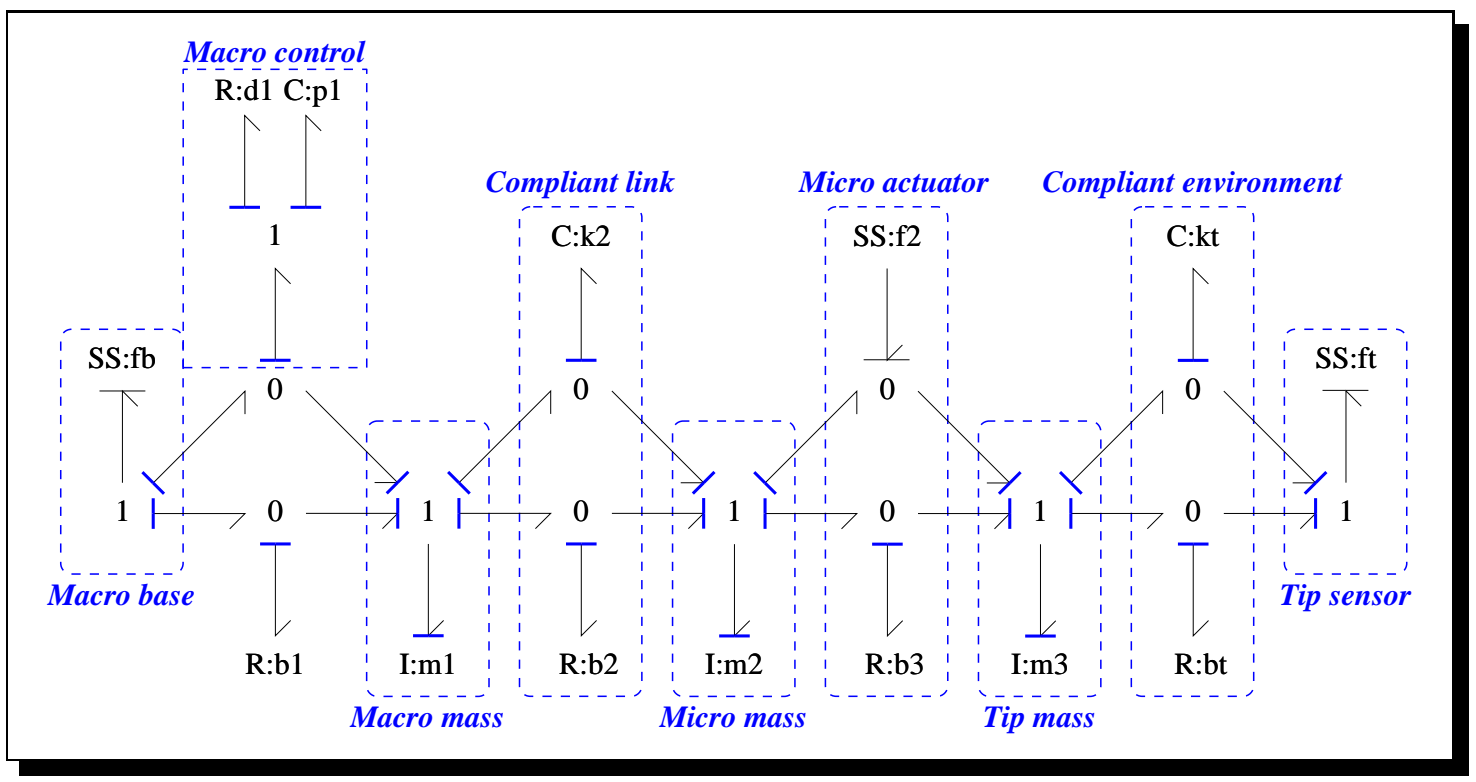

Fig. 10. Micro-macro Manipulator: Bond graph

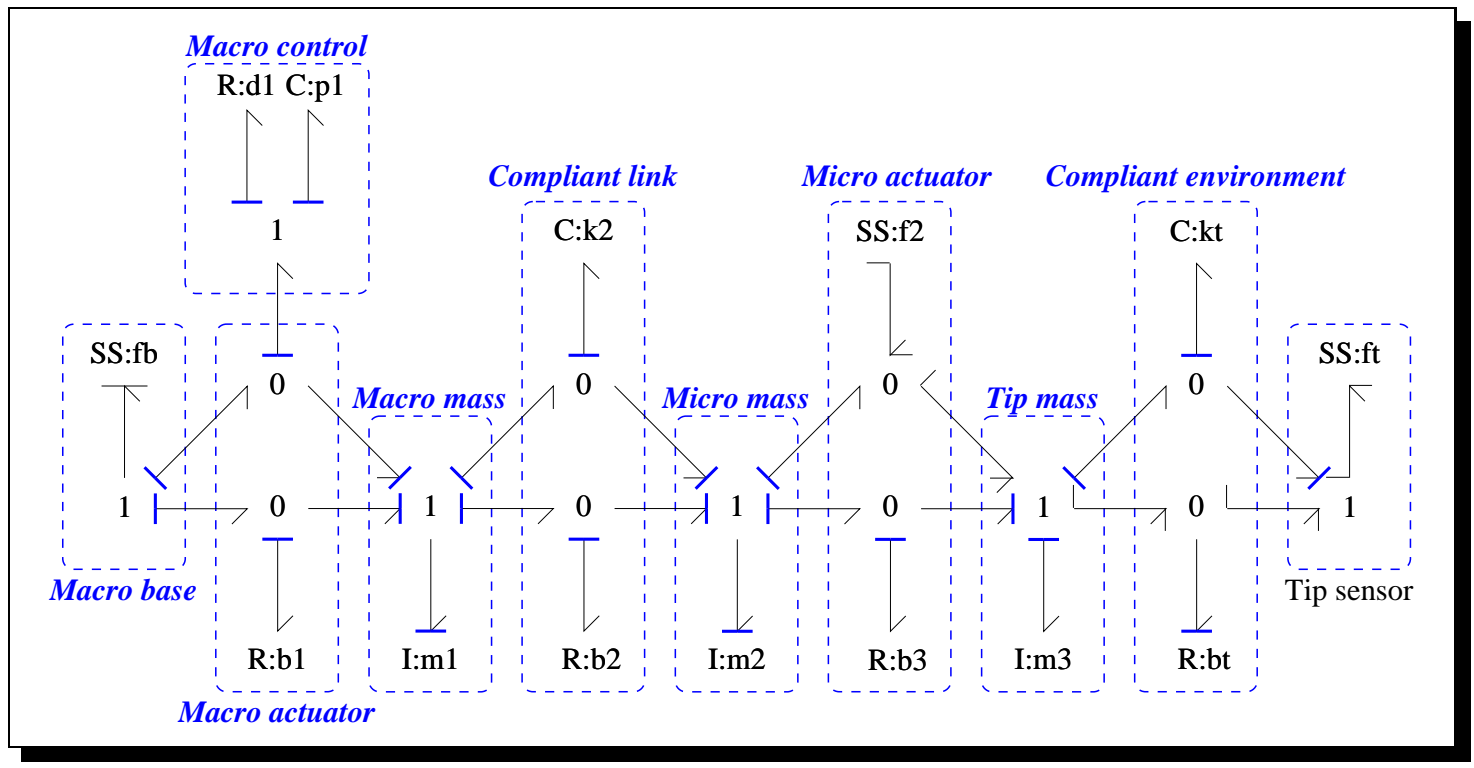

Fig. 11. Micro-macro Manipulator: Bond graph of inverse

In his PhD Thesis Sharon (1988) uses the "Design in the Physical Domain" approach (discussed by Sharon et al. (1991)) to design the force control system for a "Macro/Micro Manipulator". This is a prototype one-dimensional, two degree of freedom translational manipulator with two main components:

- a macro manipulator responsible for gross motion at the tip of which is mounted a

- micro manipulator responsible for fine motion. 
The macro manipulator has a proportional + derivative (PD) position control; the control design issue is to achieve satisfactory force control by the micro manipulator despite structural resonances. Using the "Design in the Physical Domain" approach and the notion of impedance control (Hogan, 1985), Sharon (1988) show that there is an optimal choice of macro control which simplifies the micro control problem. Here, the same conclusion is reached, again using the "Design in the Physical Domain" approach but using the bond graph of the inverse system to yield physical insight.

Figure 10 shows the system bond graph superimposed on a schematic diagram of the dynamic system. The following features are included:

- the macro control system (represented by bond graph components - see (Gawthrop, 1995b)),

- the lumped representation of the masses, compliant link and environment,

- and the two actuators.

Having included the macro controller in the system; the remaining input/output pair is the micro-actuator force $f_{2}$ and the tip force $f_{t}$. This pair is inverted in Figure 11 to examine the properties of the inverse system relevant to the micro-controller.

Table 1

\begin{tabular}{|l|l|}
\hline Name & Physical meaning \\
\hline p1 & Macro control: prop. gain \\
m1 & Macro mass \\
k2 & macro-micro link stiffness \\
m2 & Micro mass \\
\hline d1 & Macro control: deriv. gain \\
b1 & Macro actuator damping \\
b2 & Link damping \\
\hline
\end{tabular}

Inverse dynamics components

The causal strokes corresponding to this partial system inversion appear in Figure 11. Examination of this diagram gives the following information:

- the macro controller - in its bond graph representation - can also be viewed in the physical domain as a spring/damper system,

- the inverse dynamics are associated with the four dynamic components which retain integral causality (Table 1): the inverse dynamics are fourth order;

- the inverse dynamics are formed from passive components only and are therefore stable; 
- one $\mathbf{R}$ component (damping term) $d_{1}$ and one $\mathbf{C}$ component (compliance) are chosen as part of the macro control design - thus the design of the macro control loop can be used to simplify the design of the micro control loop by suitably modifying the inverse dynamics corresponding to the macro control loop.

The physical insight of Sharon (1988) was that $d_{1}$ should be chosen to absorb the maximum energy from the vibrating system forming the inverse dynamics; the design is based on the impedance matching methods of Hogan (1985). This gives inverse dynamics with the maximum damping factor.

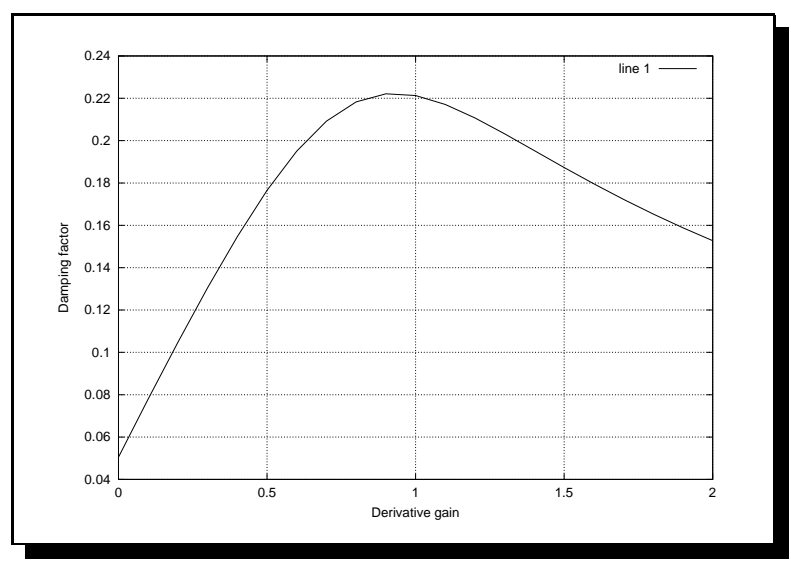

Fig. 12. Damping factor of inverse dynamics versus $d_{1}$

The qualitative insight can be turned into quantitative design by generating the inverse dynamics from the bond graph and plotting the resulting (minimum) damping factor as $d_{1}$ varies: this is done in Figure 12. It can be seen that the maximum damping of 0.29 occurs when $d_{1}=0.9$. (As discussed by Sharon (1988), the proportional gain has little effect; it is chosen to be 10 here.)

\section{Example: A System with Unstable Inverse}

A standard example of a system with an unstable inverse arises from the parallel combination of two first-order systems: one slow, with large steadystate gain and the other faster, with a smaller steady-state gain. The output of the overall system is the difference in the outputs of the two subsystems. In transfer function terms, an example of such a systems is:

$$
\begin{aligned}
G(s) & =\frac{1}{1+2 s}-\frac{1}{2+s} \\
& =\frac{1-s}{(1+2 s)(2+s)}
\end{aligned}
$$




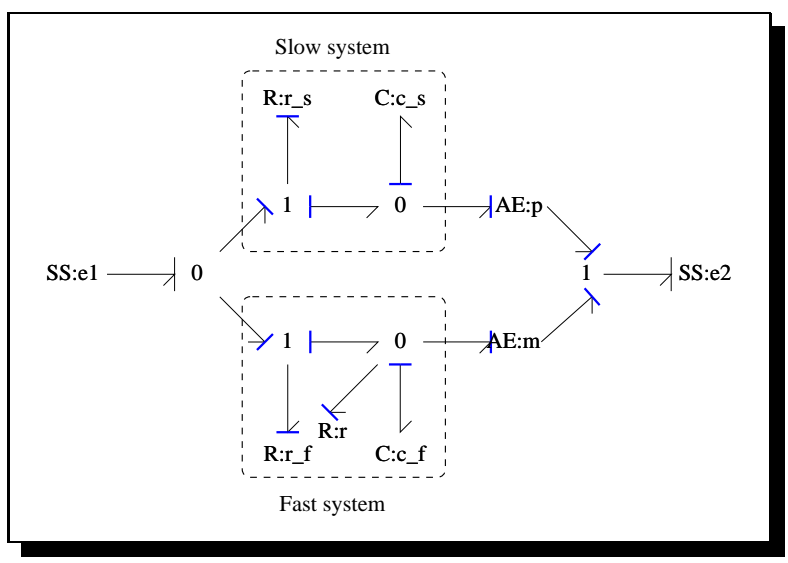

Fig. 13. System with unstable inverse: Bond graph of system

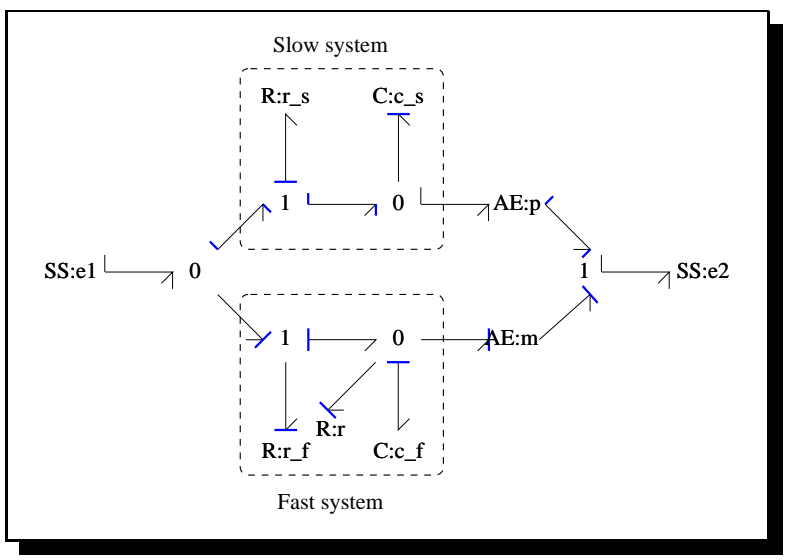

Fig. 14. System with unstable inverse: Bond graph of inverse

Although $G(s)$ is stable (with poles at $s=-2$ and $s=-0.5$ ), the inverse of this system has a pole at $s=1$ and is therefore unstable.

The bond graph of such a system appears in Figure 13. The two effort amplifier AE components and the $\mathbf{1}$ junction perform the subtraction. The SS component $\mathbf{e} 1$ provides the effort input $e_{1}$ to the system whilst SS component e2 measures the effort output $e_{2}$ as well as providing a zero flow. All components except $\mathbf{c} 2$ have unit constitutive relationship; $\mathbf{c} 2$ has a linear constitutive relationship with gain 2 .

The bond graph of the inverse system appears in Figure 14. The SS component e1 now measures the effort output (of the inverse system) $e_{1}$ of the system whilst $\mathbf{S S}$ component $\mathbf{e} \mathbf{2}$ provides the effort input (of the inverse system) $e_{2}$ as well as continuing to provide a zero flow. It is not possible to complete causality with integral causality on both $\mathbf{C}$ components; either can have derivative causality. In Figure 14, the faster system has been chosen to have derivative causality.

Comparison of Figures 13 and 14 give the following qualitative information. 
- The relative degree $\rho$ of the system is one;

- the system has one zero;

- the inverse dynamics comprise the "slow" system (with integral causality), with a positive feedback loop through the inverted "fast system".

Quantitative information is needed to determine whether or not the positive feedback loop leads to instability of the inverse system; but the purely qualitative information raises the possibility of such instability and prompts further quantitative analysis.

Using the given numerical values, the bond graph equations become, in transfer function form:

$$
\begin{aligned}
e_{s} & =\frac{1}{2 s+1} e_{1} \\
e_{1} & =(2+s)\left(e_{2}+e_{s}\right)
\end{aligned}
$$

where $e_{s}$ is the output of the slow system. Eliminating $e_{s}$ from Equations 9 and 10 gives the inverse system as

$$
e_{1}=\frac{(2 s+1)(s+2)}{1-s} e_{2}
$$

That is, the positive feedback loop does indeed lead to instability for the particular numerical values used.

This positive feedback loop mechanism for unstable inverse dynamics appears again in the Example of Section 7.

\section{Nonlinear Example: Chemical Reactor}

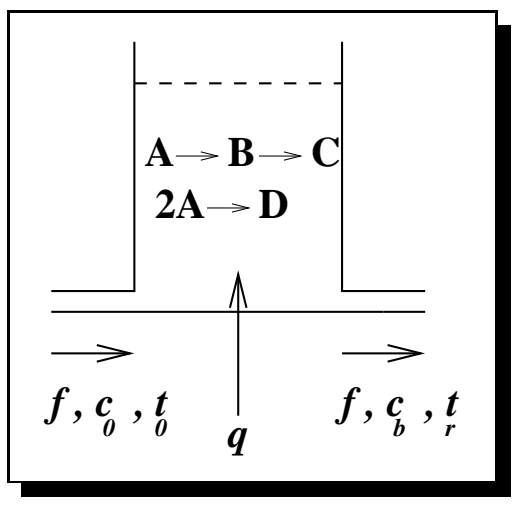

Fig. 15. Chemical reactor: Schematic

An example of a nonlinear, unstable chemical reactor with unstable zero dynamics (due to Trickett and Bogle (Tricket, 1994)) is used in this section. The 
schematic diagram appears in Figure 15 The reactor has two reaction mechanisms: $\mathrm{A} \rightarrow \mathrm{B} \rightarrow \mathrm{C}$ and $2 \mathrm{~A} \rightarrow \mathrm{D}$. The reactor mass inflow and outflow $f$ are identical. $q$ represents the heat inflow to the reactor. Following Trickett and Bogle (Tricket, 1994), the consequences of two possible choices of the two system outputs are examined

(1) either $c_{a}$ (the concentration of substance A)

(2) or $c_{b}$ (the concentration of substance B)

together with $t$, the reactor temperature. In each case, the two controlled inputs are chosen as $q$ the heat input and $f$, the reactor flow. Other (noncontrolled) inputs are $t_{0}$, the inflow temperature and $c_{0}$, the inflow concentration of substance A.

\subsection{The bond graph model}

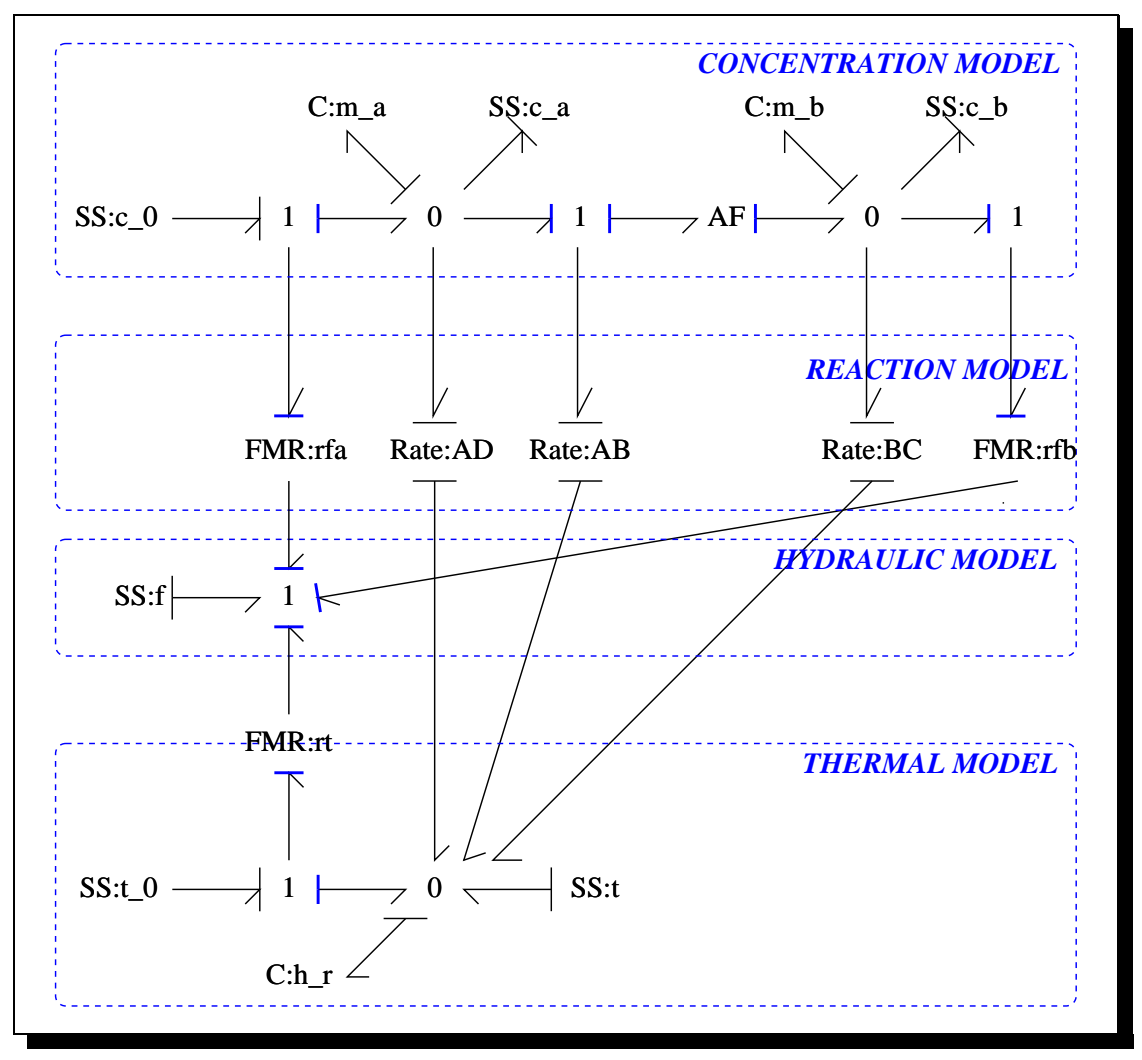

Fig. 16. Chemical reactor: Bond graph

This system was modelled using the pseudo bond graph methods of MacKenzie et al. (1993) and Gawthrop and Smith (1996). The corresponding bond graph appears in Figure 16. The bond graph components are given in Table 2. The (pseudo) bonds carry the effort/flow pairs listed in Table 3. 


\begin{tabular}{|l|l|l|}
\hline Type & label & physical meaning \\
\hline C & h_r & accumulation of enthalpy \\
C & m_a & accumulation of substance a \\
C & m_b & accumulation of substance b \\
FMR & rfa & Converts concentration $c_{0}$ to flow rate $\left(c_{0} f\right)$ \\
FMR & rfb & Converts concentration $c_{b}$ to flow rate $\left(c_{b} f\right)$ \\
FMR & rt & Converts temperature $t_{0}$ to heat flow rate $(t f)$ \\
Rate & AB & The A $\rightarrow$ B reaction kinetics \\
Rate & AD & The 2 A $\rightarrow$ D reaction kinetics \\
Rate & BC & The B $\rightarrow$ C reaction kinetics \\
SS & c_0 & inlet concentration of substance a $\left(c_{0}\right)$ \\
SS & c_a & concentration of substance a $\left(c_{a}\right)$ \\
SS & c_b & concentration of substance b $\left(c_{b}\right)$ \\
SS & f & flow rate $(f)$ \\
SS & t & temperature $(t) \&$ heat flow rate $(q)$ \\
SS & t_0 & inlet temperature $\left(t_{0}\right)$ \\
\hline
\end{tabular}

Table 2

Reactor bond graph components

Table 3

\begin{tabular}{|l|l|l|}
\hline Domain & Effort & Flow \\
\hline Concentration model & Concentration & Substance flow-rate \\
Hydraulic model & Pressure & Liquid flow-rate \\
Thermal model & Temperature & Enthalpy flow rate \\
\hline
\end{tabular}

Reactor effort/flow pairs

After normalisation, the (nonlinear) reactor equations are:

$$
\begin{gathered}
\dot{x}_{1}=-\left(\epsilon_{3} k_{3} x_{1}+u_{1}+\epsilon_{1} k_{1}\right) x_{1}+c_{0} u_{1} \\
\dot{x}_{2}=-\left(\epsilon_{2} k_{2}+u_{1}\right) x_{2}-\epsilon_{1} k_{1} x_{1} \\
\dot{x}_{3}=x_{1}^{2} \epsilon_{3} h_{3} k_{3}+x_{1} \epsilon_{1} h_{1} k_{1}+x_{2} c_{p} \epsilon_{2} h_{2} k_{2}+u_{1} t_{0}+u_{2}-\frac{x_{3}}{c_{p}} u_{1} \\
y_{1}=c_{b}=x_{2} \\
y_{2}=t=\frac{x_{3}}{c_{p}}
\end{gathered}
$$

where $u_{1}=f, u_{2}=q$ and $\epsilon_{i}=e^{-\frac{q_{i}}{t}}=e^{-\frac{q_{i} c_{p}}{x_{3}}} \cdot k_{i}$ is a rate constant and $h_{i}$ the 


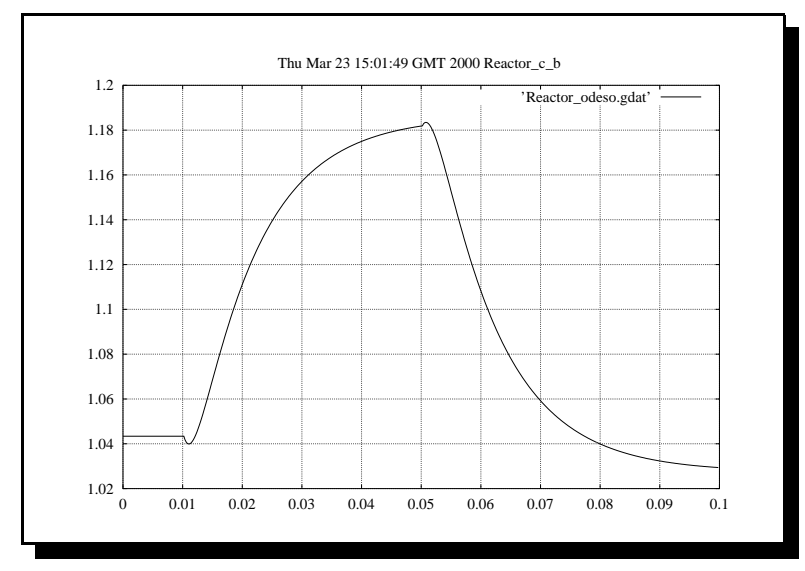

(a) Effect on $c_{b}$ of a step change in $f$

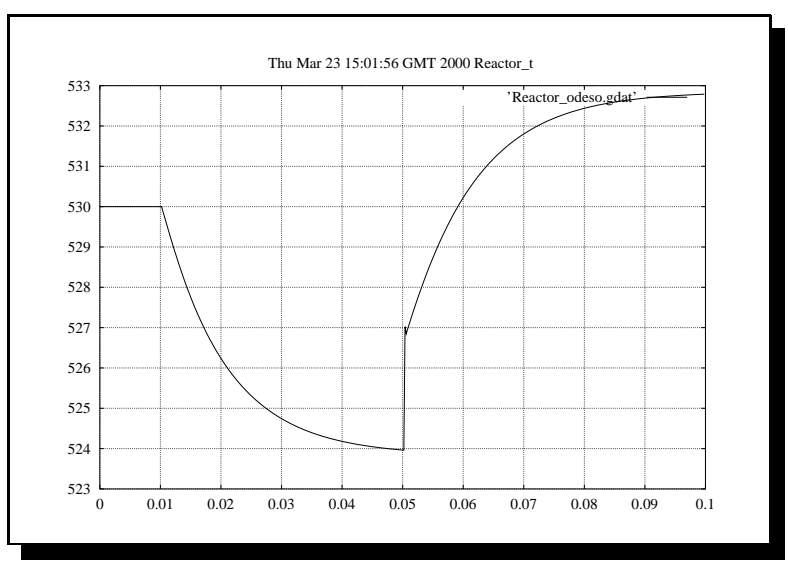

(b) Effect on $t$ of a step change in $f$

Fig. 17. Simulation of the non-linear reactor

corresponding heat of reaction. These equations could, indeed, be analysed to deduce various controllability properties as, for example by Tricket (1994). As an illustration of an approach based on equations (as opposed to the physical model), Figure 17 shows the effect on $c_{b}$ and $t$ of increasing the flow from a steady-state condition of $f=100$ and $T=530$ by $10 \%$ at time 0.01 followed by a $10 \%$ increase in the steady-state power input at time 0.05 . The initial negative-going response of $c_{b}$ suggests an unstable inverse; but, in general, a particular simulation is a poor guide to general system properties. This emphasises the main point of this paper which is to deduce system properties from the bond graph without resorting to simulation.

An alternative numerical approach is to linearise the system equations 12 and 13 about a steady state and look at the corresponding system transfer function. For example, using the steady-state corresponding to $t=t_{s}=530$ and $f=f_{s}$. Figure 18 shows the single transmission zero of the linearised system for all 


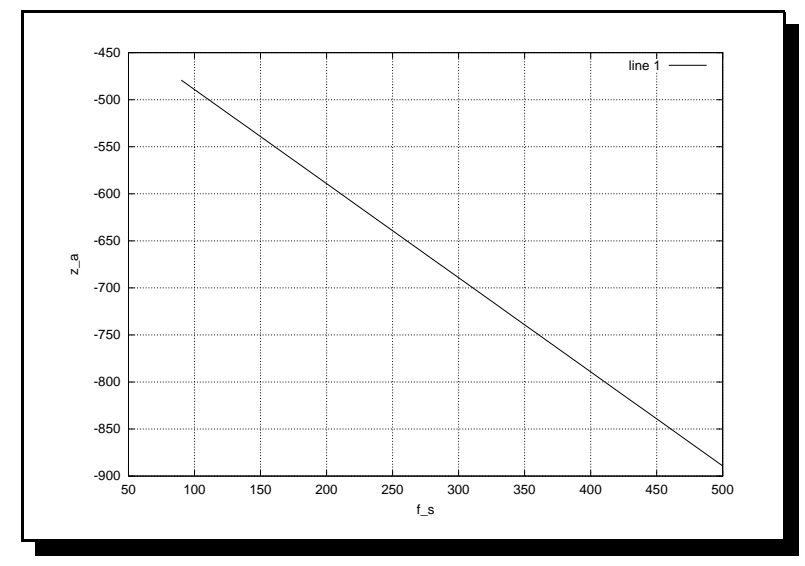

(a) With $c_{a}$ and $t$ as outputs

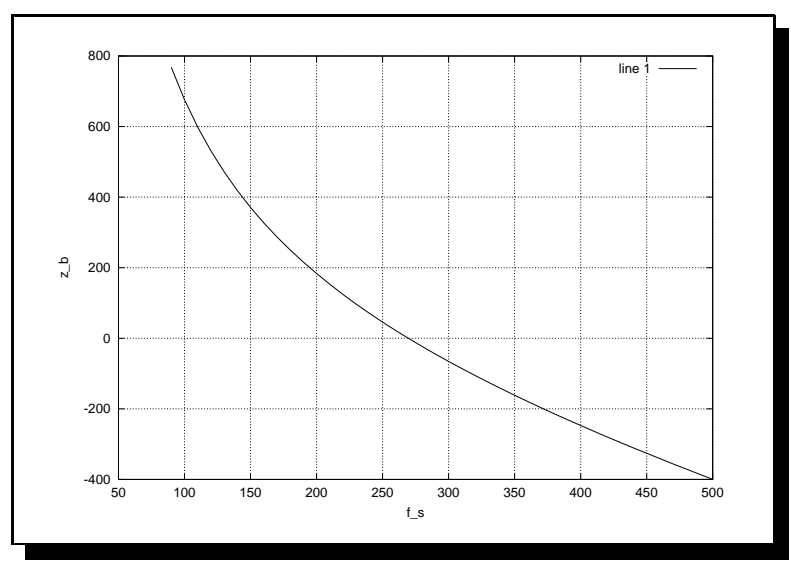

(b) With $c_{b}$ and $t$ as outputs

Fig. 18. Reactor model: Zero v. steady state flow $f_{s}$

of the values of $90 \leq f_{s} \leq 500$ for the two versions of the system. It can be seen that this corresponds to a stable inverse (negative real zero) when $c_{a}$ is used as the output but when $c_{b}$ is used as the output, the inverse is unstable for low flows. Similar analysis shows that the linearised system has two stable, and one unstable pole over this range of flow rates.

In both cases, these numerical analyses are for specific numerical values and there is no direct physical insight into the genesis of the system zeros. This is the motivation for the bond graph based analysis of the following sections. 
Table 4

\begin{tabular}{|l|l|l|l|l|l|}
\hline Section & Outputs & $\begin{array}{l}\text { Loop } \\
\text { Pair }\end{array}$ & $\begin{array}{l}\text { Other } \\
\text { Loop }\end{array}$ & $\rho$ & $m$ \\
\hline 7.2 .1 & $c_{b}, t$ & $t / q$ & open & 1 & 2 \\
7.2 .2 & $c_{b}, t$ & $t / f$ & open & 1 & 2 \\
7.2 .3 & $c_{b}, t$ & $c_{b} / f$ & closed & 1 & 1 \\
7.3 .1 & $c_{a}, t$ & $c_{a} / f$ & closed & 1 & 1 \\
\hline
\end{tabular}

Loop-pairing summary

\subsection{Bond graph analysis: $c_{b}$ and $t$ as output}

From Figure 16, it follows that the system itself has three states associated with the three $\mathbf{C}$ components. The control inputs $f$ (the flow input) and $q$ (the heat input) and two outputs ( $c_{a}$ or $c_{b}$ and $t$ in this case) are associated with the corresponding SS components. The problem is to determine the qualitative properties of the controllability of the system with respect to the possible input/output pairings.

Table 4 summarises some of the possible pairings and their controllability properties, which are discussed in the following sections. The column headed "Other loop" refers to the status of other control loop in the system it is either open-loop (no control) or closed with inversion of the other loop ("perfect" control); $\rho$ is the system relative order and $m$ the order of the inverse dynamics. The following sections illustrate the role of bond graphs in giving a "physical-domain" interpretation of the system controllability properties outlines in Section 2.

\subsection{1 t/q control loop (other loop open)}

This subsection looks at the controllability of the control-loop corresponding to the input-output pair $q$ and $t$ when the other loop $\left(f / c_{b}\right)$ is open. Referring to Figure 19, the input-output pair $f$ and $t$ are colocated and so inversion just involves moving the causal stroke on the SS component labeled $t$ to indicate that $t$ is an input and $q$ the corresponding output; the $\mathbf{C}$ component labeled $h_{r}$ now gets derivative causality. The remaining two $\mathbf{C}$ components retain integral causality. This leads to the following conclusions

- The relative order $\rho$ is one;

- The inverse dynamics have order two.

The qualitative result is that the system corresponding to the zero dynamics of this loop is second order and corresponds to the two concentration accumu- 


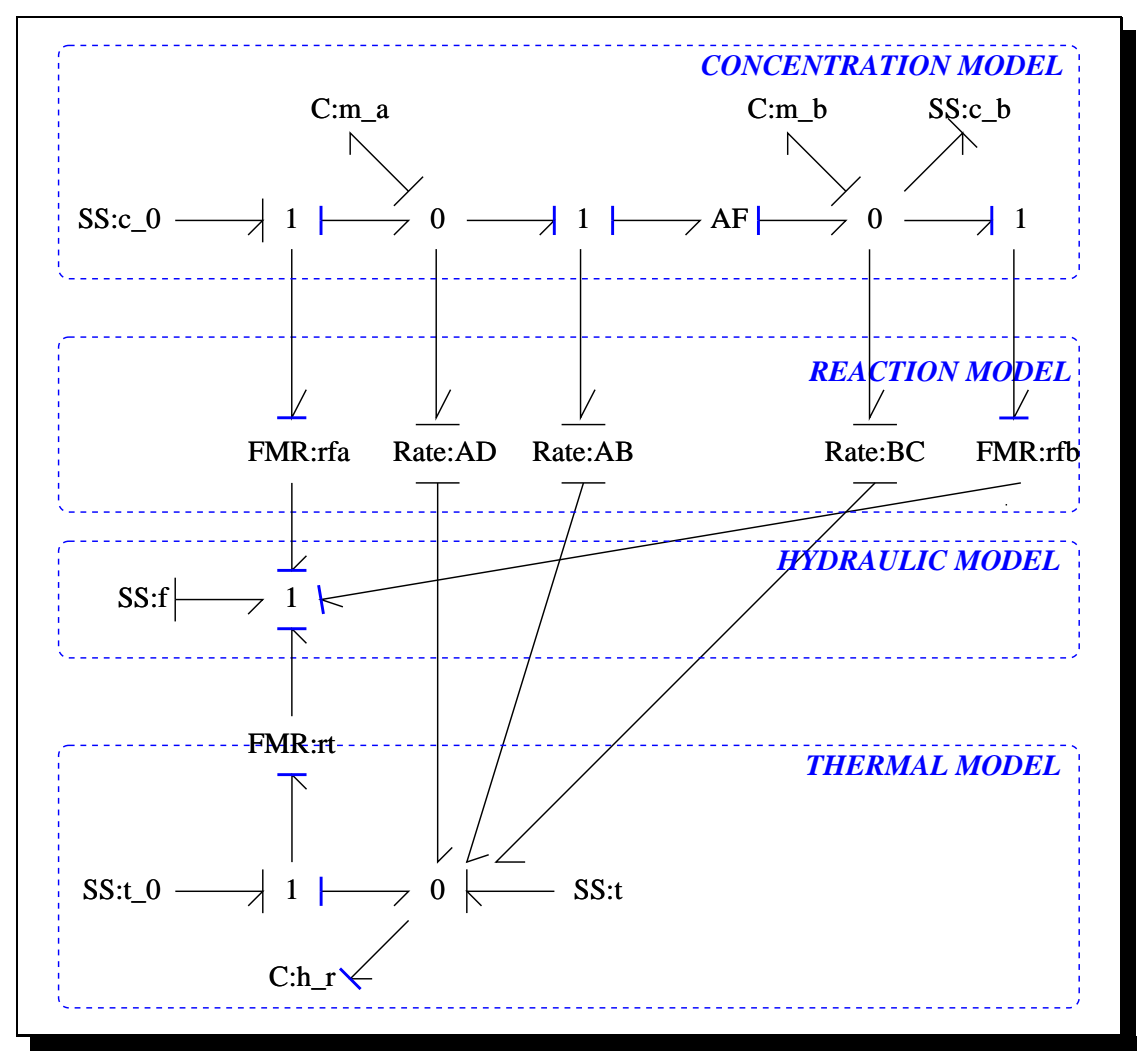

Fig. 19. Bond graph of inverse: $t / q$ (other loop open)

lating $\mathbf{C}$ components labeled c_a and c_b. Because this is a pseudo bond graph it is not possible to deduce stability from passivity considerations. However, attention is focussed on the physical dynamic system giving rise to the inverse dynamics.

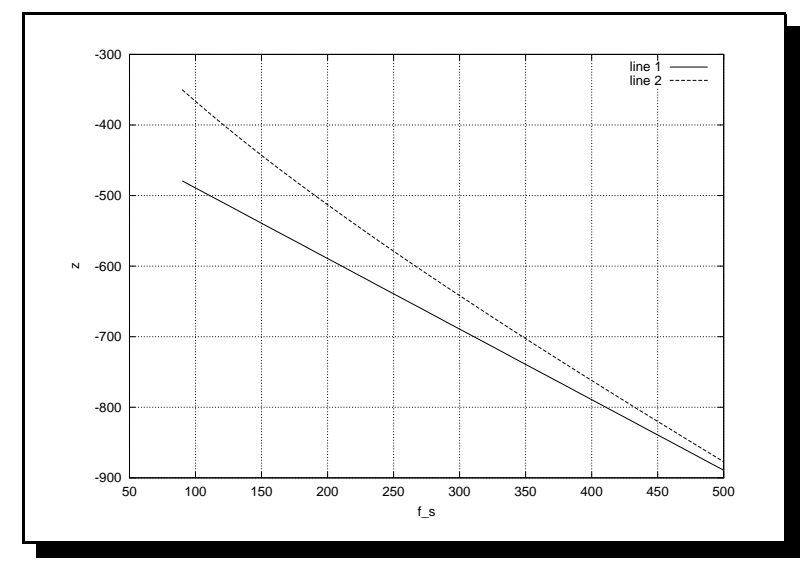

Fig. 20. Loop $t / q$ with loop $c_{b} / f$ open: Zeros v. steady state flow $f_{s}$

Directed by this qualitative analysis, the nonlinear dynamic system corresponding this partial inverse was derived from the bond graph and linearised about the steady-state corresponding to $t=t_{s}=530$ and $f=f_{s}$. Figure 20 shows the two eigenvalues of the linearised system matrix plotted against $f_{s}$; 
these are negative and real and correspond to a stable linearised system for all of the values of $90 \leq f_{s} \leq 500$.

\subsection{2 $t / f$ control loop (other loop open)}

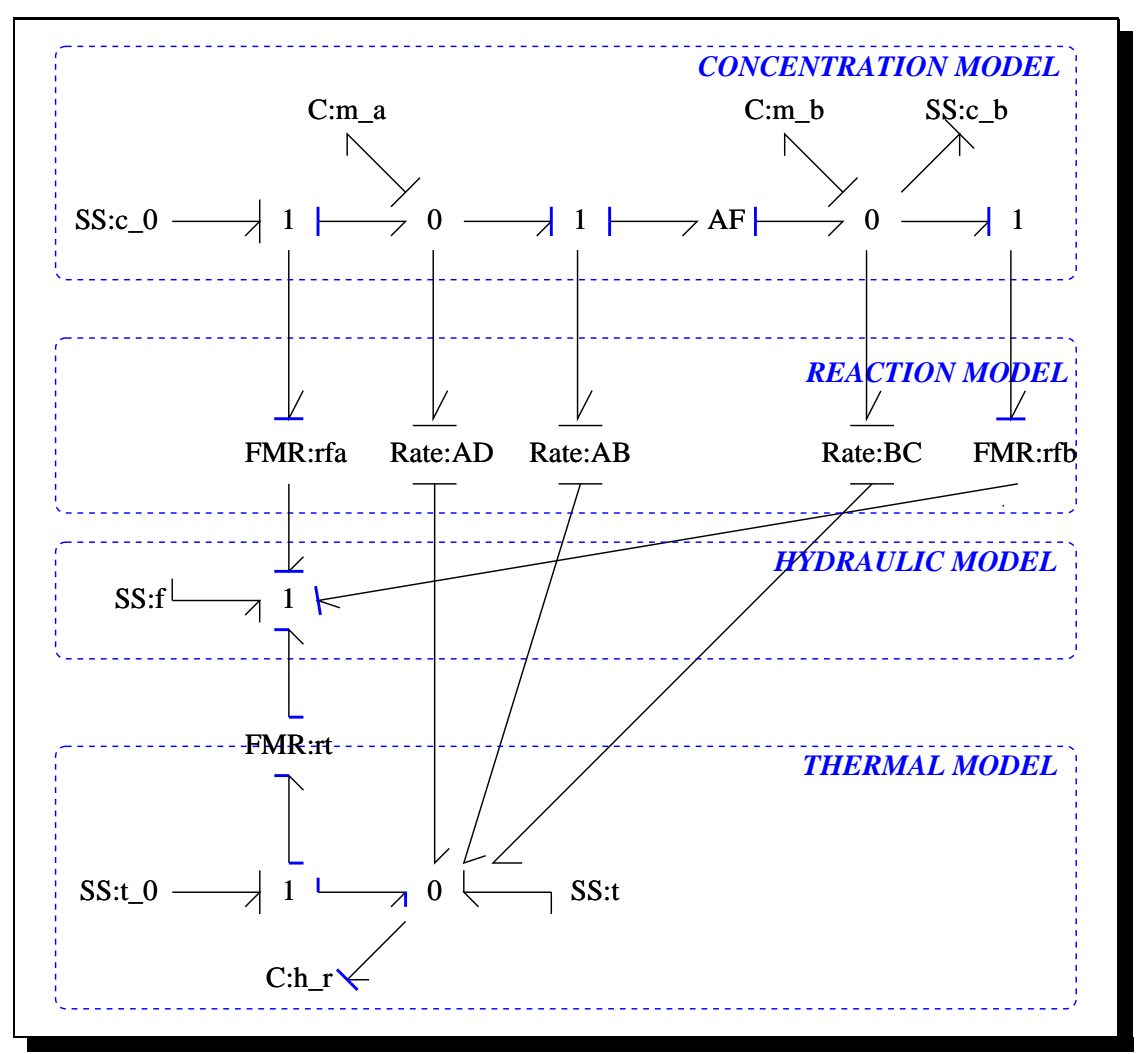

Fig. 21. Bond graph of inverse: $t / f$ (open)

This subsection looks at the controllability of the control-loop corresponding to the input-output pair $f$ and $t$ when the other loop $\left(q / c_{b}\right)$ is open. Referring to Figure 21, the input-output pair $f$ and $t$ are not colocated and so inversion involves moving the effort bicausal stroke on the SS component labeled $t$ to indicate that $t$ is an input; the corresponding flow bicausal stroke on the SS component labeled $f$ indicates that $f$ is the corresponding output. The C component labeled $h_{r}$ now gets derivative causality. The remaining two C components retain integral causality. This leads to the same qualitative conclusions as in Section 7.2.1.

Directed by this qualitative analysis, the nonlinear dynamic system corresponding this partial inverse was derived from the bond graph, it is to complicated to show here.

When the system equations are linearised about the steady-state corresponding to $t=t_{s}=530$ and $f=f_{s}$, Figure 22 shows the two eigenvalues of the linearised system matrix plotted against $f_{s}$; one of these is positive and real 


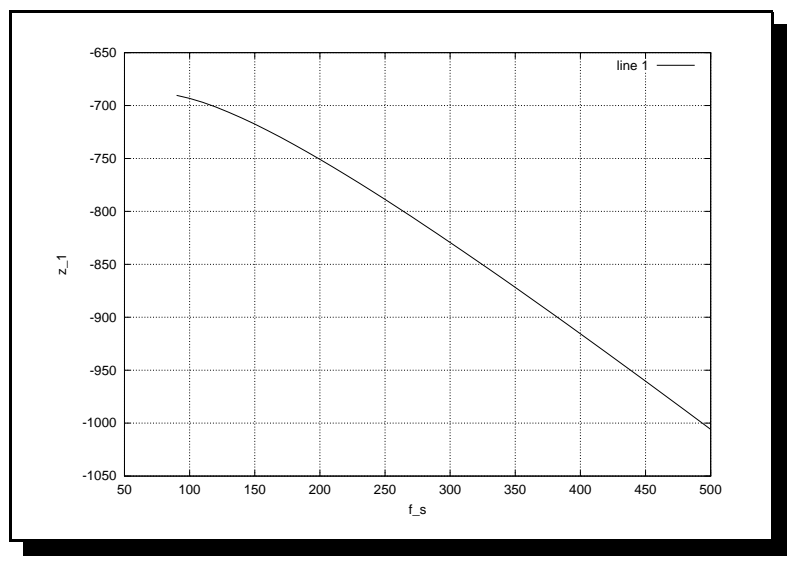

(a) Zero 1 v. steady state flow $f_{s}$

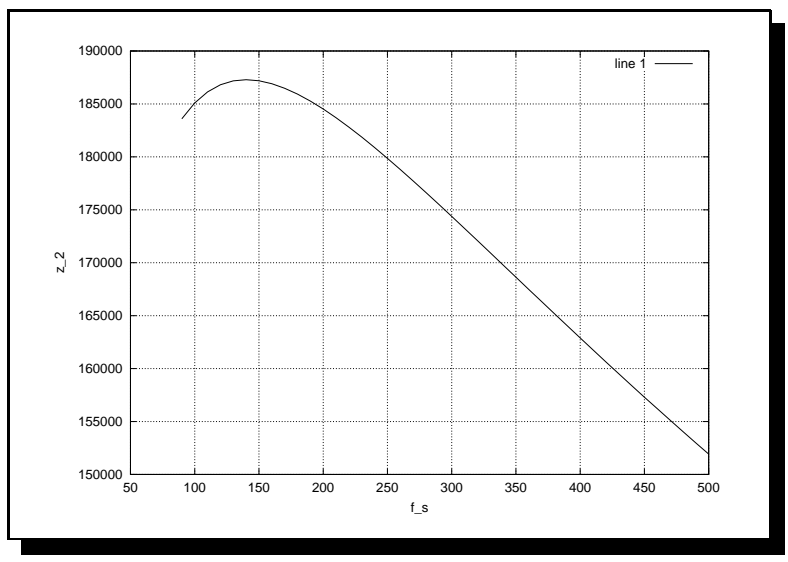

(b) Zero 2 v. steady state flow $f_{s}$

Fig. 22. Loop $t / f$ with loop $c_{b} / q$ open: Zeros v. steady state flow $f_{s}$

and corresponds to a unstable linearised inverse system for all of the values of $90 \leq f_{s} \leq 500$.

Thus, in this case, the loop is hard to control due to the unstable zero dynamics.

\subsection{3 $c_{b} / f$ control loop (other loop closed)}

This subsection looks at the controllability of the control-loop corresponding to the input-output pair $f$ and $c_{b}$ when the other $(t / q)$ is closed by by an "ideal" (inverting) controller. From section 7.2.1, it is known that it is easy to control the $t / q$ loop.

Referring to Figure 23, and comparing to Figure 16, the $t / q$ loop is inverted 


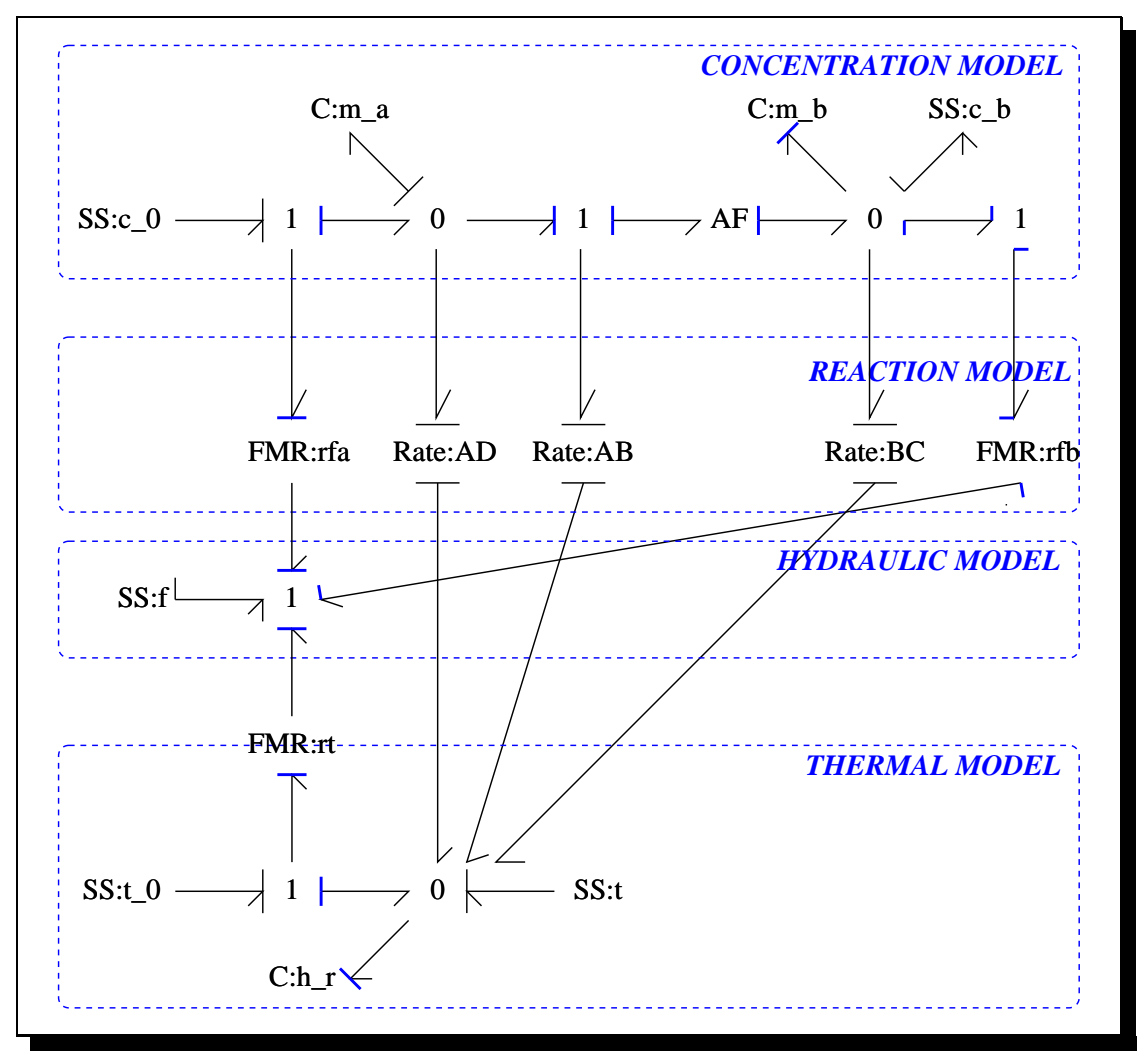

Fig. 23. Bond graph of inverse: $c_{b} / f$ (closed)

by moving the causal stroke on the corresponding SS component. The $c_{b} / f$ loop involves non-collocation and thus its inversion requires bicausal bonds.

One $\mathbf{C}$ component (h_r) was already in derivative causality due to the $t / q$ loop; thus, before inversion of the $c_{b} / f$ loop, there are two $\mathbf{C}$ components (c_a and $c_{-} b$ ) in integral causality. Inversion of the $c_{b} / f$ loop imposes derivative causality on c_b. This leads to the following conclusions

- The relative order $\rho$ is one;

- The inverse dynamics are first order.

An important feature of Figure 21 is the positive feedback loop formed via the bicausal bond from FMR:rfb to the adjacent 1 junction. As discussed in Section 6, This positive feedback loop has the potential for causing the inverse system to be unstable.

The inverse system equation is:

$$
\dot{x}_{1}=\frac{-\left(\left(c_{0}-x_{1}\right) \epsilon_{2} k_{2}+\epsilon_{3} k_{3} x_{1}^{2}\right) c_{b}+\left(c_{b}+x_{1}-c_{0}\right) \epsilon_{1} k_{1} x_{1}}{c_{b}}
$$

To investigate this (nonlinear) system further, the pole of the linearised inverse system (the zero of the linearised system) may be plotted for a constant 
$t=t_{s}=530$ and for a steady-state $f=f_{s}$ from 90 to 500 . The result is identical to that shown in Figure 18(b) corresponding to the fact that the pole of the linearised inverse system is identical to the transmission zero of the corresponding linearised (non-inverse) system. It can be seen that this zero is positive (indicating instability) for low $\left(f_{s}<260\right)$ steady-state flows.

\subsection{Bond graph analysis: $c_{a}$ and $t$ as output}

For comparison with Section 7.2, this section looks at the case where $c_{a}$ replaces $c_{b}$ as the second output variable. Rather that repeat all the details, the equivalent of Section 7.2.3 is analysed.

\subsection{1 $c_{a} / f$ control loop (other loop closed)}

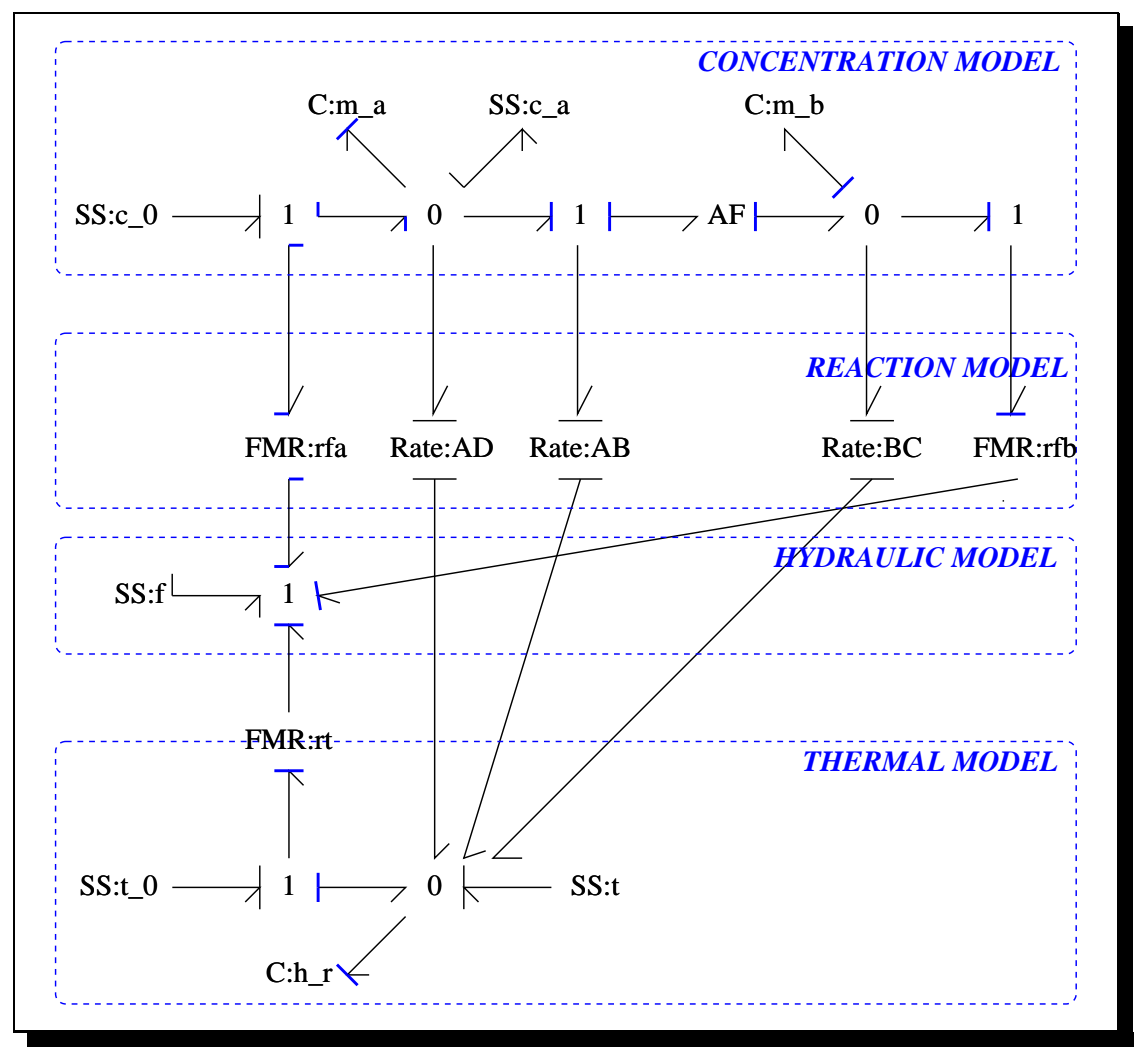

Fig. 24. Bond graph of inverse: $c_{a} / f$ (other loop closed)

This subsection looks at the controllability of the control-loop corresponding to the input-output pair $f$ and $c_{a}$ when the other $(t / q)$ is closed by by an "ideal" (inverting) controller. From section 7.2.1, it is known that it is easy to control the $t / q$ loop.

Referring to Figure 24, and comparing to Figure 16, the $t / q$ loop is inverted 
by moving the causal stroke on the corresponding SS component. The $c_{a} / f$ loop involves non-collocation and thus its inversion requires bicausal bonds.

One $\mathbf{C}$ component ( $\mathrm{h} \_\mathrm{r}$ ) was already in derivative causality due to the $t / q$ loop; thus, before inversion of the $c_{a} / f$ loop, there are two $\mathbf{C}$ components (c_a and $c_{-} b$ ) in integral causality. Inversion of the $c_{a} / f$ loop imposes derivative causality on c_a. This leads to the following conclusions

- The relative order $\rho$ is one;

- The inverse dynamics are first order.

Unlike the system of Section 7.2.3, there is no bicausal loop present.

The differential equation describing the inverse dynamics is:

$$
\dot{x}_{1}=\frac{-\left(\left(c_{0}-c_{a}\right) \epsilon_{2} k_{2}+c_{a}^{2} \epsilon_{3} k_{3}\right) x_{1}+\left(c_{a}+x_{1}-c_{0}\right) c_{a} \epsilon_{1} k_{1}}{c_{0}-c_{a}}
$$

Once again, the pole of the system linearised as in Section 7.2.3 may be plotted and, in a similar fashion, the result is identical to that shown in Figure 18(a). However, unlike the result of Section 7.2.3 corresponding to Figure 18(b), this indicates that the linearised system has stable inverse dynamics over the range of linearisation.

\section{Conclusion}

It has been shown, and illustrated by three examples, that bond graphs (when augmented by the bicausality concept) provide a powerful approach to examining the controllability properties of a dynamic system, expressed in terms of the system zeros, directly from the system bond graph.

In particular, the bicausality concept allows the bond graph of the inverse to be deduced directly from that of the system itself; examination of this bond graph gives information about the system zeros (in the linear case) and system zero dynamics (in the nonlinear case). This enables the control engineer to relate the abstract concept of system zeros to the physical model encapsulated in the system bond graph. One aspect of this insight is that a physical explanation of unstable inverse dynamics has been given in terms of a positive feedback loop caused by the corresponding inversion.

In some cases, the stability of the inverse dynamics is dependent on the numerical values of system parameters and thus a purely qualitative approach is insufficient. However, as shown in the Examples, the qualitative approach 
focuses attention on the parts of the system giving rise to zero dynamics and guides the numerical computations required.

In a multiloop context (as illustrated in Sections 5 and 7), the controller applied to each loop affects the dynamics of the other loop. In Section 5, it was shown how the explicit design of a PD controller could be used to help the design of the other loop controller. In contrast, Section 7 looked at two extreme cases for the control of the other loop; open and "ideal"; this is related to recent work on controllability indicators for linear two-loop systems (Gawthrop et al., 1999).

As such, the paper makes a contribution to the design of control systems in the physical domain.

\section{Acknowledgements}

I would like to acknowledge discussions at the Department of Chemical Engineering of Edinburgh University with Murray Laing on controllability and Jack Ponton on systems with inverse response. The reactor problem arose partly from discussions with Des Costello (same department) and partly from a seminar given by David Bogle of University College, London, when visiting Edinburgh.

I am grateful to the two anonymous referees for their detailed comments and suggestions which have improved the paper substantially.

\section{References}

Bode, H. W. (1945). Network Analysis and Feedback Amplifier Design. van Nostrand. New York.

Bristol, E. H. (1966). On a new measure of interactions for multivariable process control. IEEE Trans. on Automatic Control AC-11, 133-134.

Cellier, F. E. and Granda, J. J., Eds.) (1995). Proceedings of the 1995 International Conference On Bond Graph Modeling and Simulation (ICBGM'95). Vol. 27 of Simulation Series. Society for Computer Simulation. Las Vegas, U.S.A.

Cornet, A. and F. Lorenz (1989). Equation ordering using bond graph causality analysis. In: Modelling and Simulation of Systems (P. Breedveld et. al, Ed.). IMACS. pp. 55-58.

Douglas, J. M. (1988). Conceptual Design of Chemical Processes. McGraw-Hill. 
Gawthrop, P. J. (1995a). Bicausal bond graphs. In: Cellier and Granda (1995). pp. 83-88.

Gawthrop, P. J. (1995b). Physical model-based control: A bond graph approach. Journal of the Franklin Institute.

Gawthrop, P. J. and L. P. S. Smith (1996). Metamodelling: Bond Graphs and Dynamic Systems. Prentice Hall. Hemel Hempstead, Herts, England.

Gawthrop, P. J. and L. Smith (1992). Causal augmentation of bond graphs. Journal of the Franklin Institute 329(2), 291-303.

Gawthrop, Peter J. (1997). Control system configuration: Inversion and bicausal bond graphs. In: Granda and Dauphin-Tanguy (1997). pp. 97-102.

Gawthrop, Peter J. (1999). MTT: Model transformation tools. Online WWW Home Page. URL: http://www.eng.gla.ac.uk/ peterg/software/MTT/.

Gawthrop, Peter J., Donald J. Ballance and Genevieve Dauphin-Tanguy (1999). Controllability indicators from bond graphs. In: Proceedings of the 1999 International Conference On Bond Graph Modeling and Simulation (ICBGM'99) (J. J. Granda and F. Cellier, Eds.). Vol. 31 of Simulation Series. Society for Computer Simulation. San Francisco, California, U.S.A.. pp. 359364.

Granda, J. J. and Dauphin-Tanguy, G., Eds.) (1997). Proceedings of the 1997 International Conference On Bond Graph Modeling and Simulation (ICBGM'97). Vol. 29 of Simulation Series. Society for Computer Simulation. Phoenix, Arizona, U.S.A.

Havre, K. and S. Skogestad (1996). Input/output selection and partial control. In: Preprints of the 13th IFAC World Congress. San Francisco, California, U.S.A.

Hogan, N. (1985). Impedance control: An approach to manipulation. ASME Journal of Dynamic Systems, Measurement and Control.

Horowitz, I. M. (1963). Synthesis of Feedback Systems. Academic Press.

Huang, S. Y. and K. Youcef-Toumi (1999). Zero dynamics from bond graph models - Part I: SISO systems; Part II: MIMO systems. ASME Journal of Dynamic Systems, Measurement and Control 121(1), 10-26.

Isidori, A. (1995). Nonlinear Control Systems: An Introduction. 3rd Ed.. SpringerVerlag. New York.

Kailath, T. (1980). Linear Systems. Prentice-Hall.

Karnopp, D. C. (1979). Bond graphs in control: Physical state variables and observers. J. Franklin Institute 308(3), 221-234.

Karnopp, D. C. (1995). Actively controlled systems: - an ideal application area for bond graph modelling (plenary address). In: Cellier and Granda (1995). p. 3. 
Karnopp, D. C. and R. C. Rosenberg (1975). System Dynamics: A Unified Approach. John Wiley.

Karnopp, D. C., D. L. Margolis and R. C. Rosenberg (1990). System Dynamics: A Unified Approach. John Wiley.

Kwakernaak, H. and R. Sivan (1972). Linear Optimal Control Systems. Wiley.

Laing, D. M. (1995). Integrated Process and Control System Design. PhD thesis. Dept. Chemical Engineering, Edinburgh University.

Maciejowski, J. M. (1989). Multivariable Feedback Design. Addison-Wesley.

MacKenzie, S. A., P. J. Gawthrop and R. W. Jones (1993). Modelling chemical processes with pseudo bond graphs. In: Proceedings of the International Conference On Bond Graph Modeling (ICBGM'93) (J. J. Granda and F. E. Cellier, Eds.). Vol. 25 of Simulation Series. Society for Computer Simulation. La Jolla, California, U.S.A.. pp. 327-332.

Marino, Riccardo and Patrizio Tomei (1995). Nonlinear Control Design: Geometric, Adaptive \& Robust. Prentice Hall.

Ngwompo, R. F. (1997). Contribution au Dimensionnement des Systèms sur des Critères Dynamiques et Énergétiques - Approche par Bond Graph. PhD thesis. INSA de Lyon.

Ngwompo, R. F., S. Scavarda and D. Thomasset (1996). Inversion of linear timeinvariant siso systems modelled by bond graph. Journal of the Franklin Institute 333(B)(2), 157-174.

Ngwompo, R. F., S. Scavarda and D. Thomasset (1997). Structural invertibility and minimal inversion of multivariable linear systems - a bond graph approach. In: Granda and Dauphin-Tanguy (1997).

Ponton, J. W. and D. M. Laing (1993). A hierarchical approach to the design of process control systems. Trans. I. Chem. E. 71, 181-188.

Roberts, D. W., D. J. Ballance and P. J. Gawthrop (1995). Design and implementation of a bond graph observer for robot control. Control Engineering Practice 3(10), 1447-1457.

Rosenberg, R. C. and D. C. Karnopp (1983). Introduction to Physical System Dynamics. McGraw-Hill.

Sharon, A. (1988). The Macro/Micro Manipulator: An Improved Architecture for Robot Control. PhD thesis. MIT.

Sharon, A., N. Hogan and D. E. Hardt (1991). Controller design in the physical domain. Journal of the Franklin Institute 328(5), 697-721.

Skogestad, S. and I Postlethwaite (1996). Multivariable Feedback Control Analysis and Design. Wiley. 
Thoma, J. (1975). Introduction to Bond Graphs and their Applications. Pergamon Press.

Tricket, K. J. (1994). Quantification of Inverse Responses for Controllability Assessment of Nonlinear Processes. PhD thesis. University College London.

Wellstead, P. E. (1979). Introduction to Physical System Modelling. Academic Press.

Wolff, E. A. (1994). Studies on Control of Integrated Plants. PhD thesis. University of Trondheim. 